POS $\quad$ PROCEEDINGS

\title{
A new look at radio loud AGN unification
}

\section{Markos Georganopoulos*}

Department of Physics, Joint Center for Astrophysics, University of Maryland Baltimore County, 1000 Hilltop Circle, Baltimore, MD 21250, USA

E-mail: georgano@umbc.edu

\section{Eileen T. Meyer}

Department of Physics and Astronomy, Rice University, Houston, TX 77005

\section{Giovanni Fossati}

Department of Physics and Astronomy, Rice University, Houston, TX 77005

\section{Matthew L. Lister}

Department of Physics, Purdue University, 525 Northwestern Ave., West Lafayette, IN 47907, USA

\begin{abstract}
We review some open issues on our understanding of the radio loud AGN unification and the blazar sequence, and discuss the results of our recent work [Meyer et al.(2011)] and current and future work in the direction of developing an extension of the unification scheme. In the scheme we envisage, sources jets with accretion power greater than $\sim 10^{-2}$ of the Eddington luminosity [Narayan et al.(1997), Ghisellini et al.(2009)] have jets that emanate from the vicinity of radiatively efficient accretion disks with strong broad line regions, and the jets are characterized by a single flow velocity. Sources with accretion power less than $\sim 10^{-2}$ of the Eddington luminosity have jets that emanate from the vicinity of radiatively inefficient accretion disks with weak or no broad emission line regions, and the jet flows exhibit velocity gradients in the form of a decelerating [Georganopoulos \& Kazanas(2003)] and/or a fast spine/slow sheath flow [Ghisellini et al.(2005)]. The phenomenology of a source, therefore, depends on its accretion environment, the jet power, and the jet orientation relative to the line of sight. The situation emerging from Fermi observations is briefly discussed along with ways to evaluate this scheme through comparison to observations.
\end{abstract}

AGN Physics in the CTA Era-AGN2011,

May 16-17, 2011

Toulouse, France

\footnotetext{
*Speaker.
} 


\section{Open problems in our understanding of radio loud AGN}

It is by now clear that most galaxies host supermassive black holes (SMBH; $M \sim 10^{6}-10^{9} M_{\odot}$, where $M_{\odot}$ is the Solar mass). When these SMBH accrete matter, they radiate copiously (assuming isotropic luminosity, up to $\sim 10^{49} \mathrm{erg} \mathrm{s}^{-1}$ ) through one or both of the following outlets: (i) IR to X-ray emission from the molecular torus, the accretion disk that forms around the black hole, and the X-ray emitting corona presumed to 'sandwich' the disk and (ii) radio to $\mathrm{TeV}$ emission from relativistic jets that form close to the black hole and propagate to distances up to $\sim \mathrm{Mpc}$ scales. The jet emission comes in two broad spectral components, one peaking in the IR to X-rays, almost certainly synchrotron emission, and one peaking at MeV-TeV energies [Böttcher(2007)], most probably inverse Compton (IC). Jets are seen in only a few percent of the active galactic nuclei (AGN). These are classified as radio loud, in the sense that the ratio of the radio (synchrotron radiation coming from the jet) to the nuclear optical (coming form the accretion disk) flux is above a certain limit [Urry \& Padovani(1995)]. These jets, once thought to be immaterial to the evolution of the host galaxy cluster, are now seen as potentially central players in providing a feedback mechanism that offsets the cooling of the cluster gas [McNamara \& Nulsen(2007)].

\subsection{We do not know how accretion and ejection are coupled}

The fraction of all AGN that, by virtue of exhibiting large scale radio jets, are classified as radio loud is only a few percent. Understanding why systems that seem to have very similar accretion disks may or may not produce relativistic jets is critical in understanding the physical mechanisms in play. Ultimately, there must be a physical parameter beyond the mass of the black hole $M$ and the accretion rate $\dot{M}$ that regulates if relativistic jets will be formed and how strong they will be. The two most popular mechanisms for jet production are based on the ability of the magnetic field that threads the accretion disk [Blandford \& Payne(1982)] or the black hole itself

[Blandford \& Znajek(1977)], provided the black hole has significant spin, to extract rotational energy from the accretion disk or the black hole and produce a collimated outflow.

The observational connection, however, between jet and accretion power, namely if for a given accretion power the jet kinetic power is fixed or if it covers a wide range of powers, remains unsettled: [Sikora et al.(2007)] found that for a given nuclear optical luminosity (a proxy for the accretion power), there are two families of AGN, radio loud, hosted mostly by elliptical galaxies and radio quiet hosted mostly by spiral and disk galaxies, with the radio luminosity of the radio loud sources being $\sim 10^{3}$ times higher that that of the radio quiet for the same nuclear optical emission. On the other hand, recent Spitzer observations [Fernandes et al.(2011)], where the 12 $\mu m$ emission was used as a proxy for the accretion power and the $151 \mathrm{MHz}$ luminosity as a proxy for the jet power, suggest that for a given accretion power there is a very wide range of jet powers, with the maximum jet power increasing with the accretion power.

\subsection{The unification of radio loud AGN and its open issues}

The power dichotomy. A key morphological separation of radio loud AGN is that between the powerful Fanaroff-Riley (FR) [Fanaroff \& Riley(1974)] type II radio galaxies (RG) with jets that remain collimated up to $\sim$ Mpc scales and terminate at hotspots, locations where the flow decelerates abruptly and inflates the radio lobes, and the weaker FR I RG with jets that decelerate and 
de-collimate to gradually dimming diffuse radio lobes. According to the orientation-based unification scheme [Urry \& Padovani(1995)], the aligned population of FR I RG are BL Lacertae objects, while the aligned population of FR II RG are Flat Spectrum Radio Quasars (FSRQ). Depending on the optical spectra, the division between the featureless BL Lac objects and quasar - like, broad emission line FSRQs, is set arbitrarily to a $5 \AA$ equivalent width (EW).

Dichotomy violations. This scenario has been recently called into question with the discovery of powerful, FR II type BL Lacs, and weak, FR I type FSRQs [Landt et al.(2006), Kharb et al.(2010)]. Likewise, the spectral classification has come under scrutiny as some BL Lacs do show intermittent broad lines [Stickel et al.(1991), Vermeulen et al.(1995), Stocke et al.(2011)], and spectroscopic work has shown that there is probably a continuum rather than bimodal of emission line strengths. [Scarpa \& Falomo(1997)].

In addition, as was pointed out originally by [Georganopoulos \& Marscher(1998)] and recently by [Ghisellini et al.(2011)], because the EW depends on both the emission line strength and the beamed non-thermal continuum, there must be sources with strong emission lines that are seen as BLs because the jet non thermal emission peaks close to the optical and dominates over the lines. Similarly, there must be sources with weaker broad emission lines that because the jet non thermal emission peaks at significantly lower than optical energies, they may reach an EW that classifies them as FSRQs. This explains qualitatively the finding of [Scarpa \& Falomo(1997)], as there is no physically justified EW range that is not feasible and a bimodal EW distribution is not expected. All of this creates serious problems for a unification scheme which connects spectral type with the morphology.

\subsection{The need for velocity gradients in weak jets}

Overluminous FR I and the spine-sheath idea. [Chiaberge et al.(2000)] and

[Trussoni et al.(2003)] showed that the FR I nuclei are overluminous by a factor of $10-10^{4}$ compared with their luminosity should they have been unaligned BLs with Lorentz factors $\Gamma=15$. These authors suggested that the flow is characterized by a fast spine-slow sheath geometry as a way to explain the persistence of emission at large angles: while at small angles the spectral energy distribution (SED) is dominated by the fast spine, at large angles the spine is beamed away from our line of sight and we observe the emission from the less beamed slow sheath. A model along these lines was presented by [Ghisellini et al.(2005)].

Decelerating jets. A fast spine, however, contradicts the lack of superluminal velocities at VLBI scales in weak BLs [Piner et al.(2010)]. These sources, however, require Lorentz factors of the order of 50 and higher for modeling their nuclear SED and avoiding pair production absorption of the gamma-rays [Georganopoulos \& Kazanas(2003), Begelman et al.(2008)]. Motivated by the above considerations, [Georganopoulos \& Kazanas(2003)] proposed that the jet flow decelerates at the sub-pc scales. They found (left panel of figure 1) that such flows can reproduce the observed trends. This is due to upstream Compton (UC) scattering, in which the low energy seed photons, produced mostly at the slow downstream part of the flow, are seen by the most energetic electrons at the fast base of the flow and are boosted due to their different bulk Lorentz factors. They also found (right panel of figure 1) that this scenario reproduces very well the luminosity differences of BLs and their unbeamed parent population FR Is [Trussoni et al.(2003)]. The two models, decelerating and spine sheath could in principle be distinguished from variability studies, in the sense that 

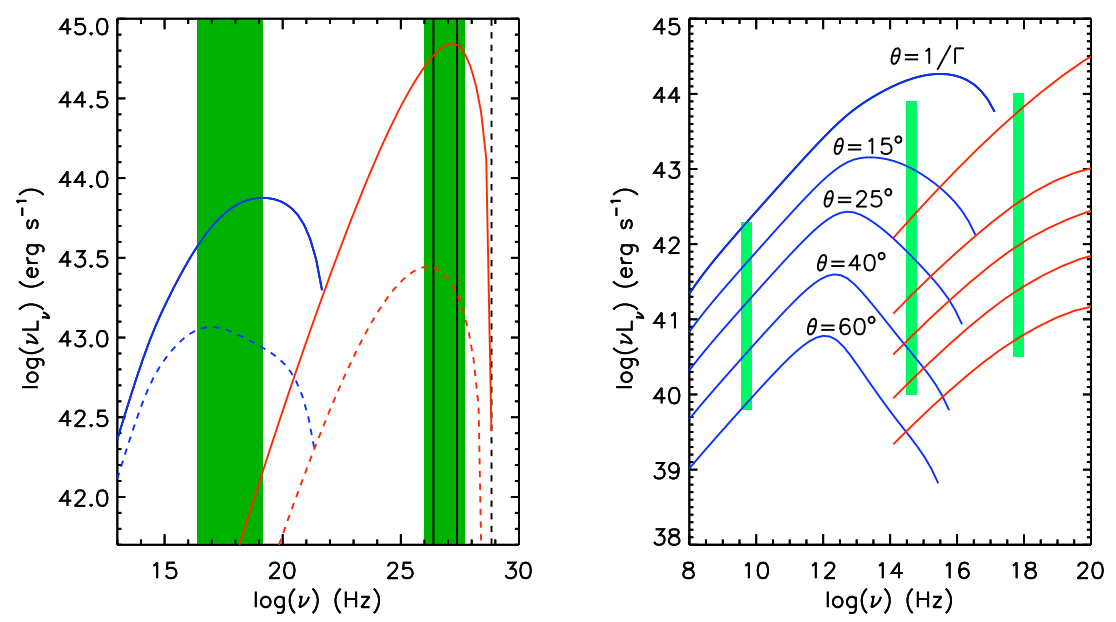

Figure 1: From [Georganopoulos \& Kazanas(2003)]. Left panel: the SED of a decelerating relativistic flow under $\theta=3^{\circ}$ (solid line) and $\theta=6^{\circ}$ (broken line) observing angles. The flow decelerates from $\Gamma_{1}=15$ to $\Gamma_{2}=4$ within $2 \times 10^{16} \mathrm{~cm}$. The green bars indicate approximately the $X$-ray and $\mathrm{TeV}$ regimes. Right panel: The SED of a decelerating flow for a range of observing angles. The green bars correspond to the average luminosity difference in radio, optical and X-rays, between the samples of BLs and FR I RG studied by [Trussoni et al.(2003)].

while the variations of the lower energies causally connected to those at higher energies in the decelerating flow, the connection between spine and sheath is not unique and different variability responses can be accomodated.

\subsection{The blazar sequence and observations that challenge it}

The sequence. In blazars, the synchrotron peak frequency $\left(v_{s}\right)$ covers a wide range (from $10^{12}$ to $\left.10^{18} \mathrm{~Hz}\right)$, with FSRQ sources having low $v_{s}\left(<10^{14.5} \mathrm{~Hz}\right)$, and BL Lacs spanning the entire range. Following [Abdo et al.(2010)], we adopt the generic terms for low, intermediate, and high synchrotron-peaking (LSP, ISP, HSP) blazars independently of the spectroscopic type. [Fossati et al.(1998)] found (see Figure 2) that as the source synchrotron power $L_{s}$ increases, $v_{s}$ decreases, with predominantly powerful FSRQ sources at the low-peak, high-luminosity end through LSP, ISP, and finally HSP BL Lacs at the low-luminosity end. They also used the sparse EGRET data to argue that the same reduction of the peak frequency happens in the high energy (presumably IC component) component and that the Compton dominance (the ratio of IC to synchrotron power) increases with source power. [Ghisellini et al.(1998)] suggested that more efficient cooling of particles in the jets of high luminosity blazars is responsible for the lower peak frequencies.

From sequence to envelope. [Padovani et al.(1997)] and [Perlman et al.(1998)] identified relatively powerful sources with a radio to X-ray spectral index $\alpha_{R X}$ typical of weak sources with $v_{s}$ in the X-rays. Such sources, if confirmed would challenge the sequence. Upon close study, however, their X-ray emission was found not to be of synchrotron origin [Landt et al.(2008)] and as of now sources with high $L_{s}$ - high $v_{s}$ have not been found [Landt et al.(2006), Padovani(2007), Landt et al.(2008), Maraschi et al.(2008)]. Sources below the blazar sequence are expected from 


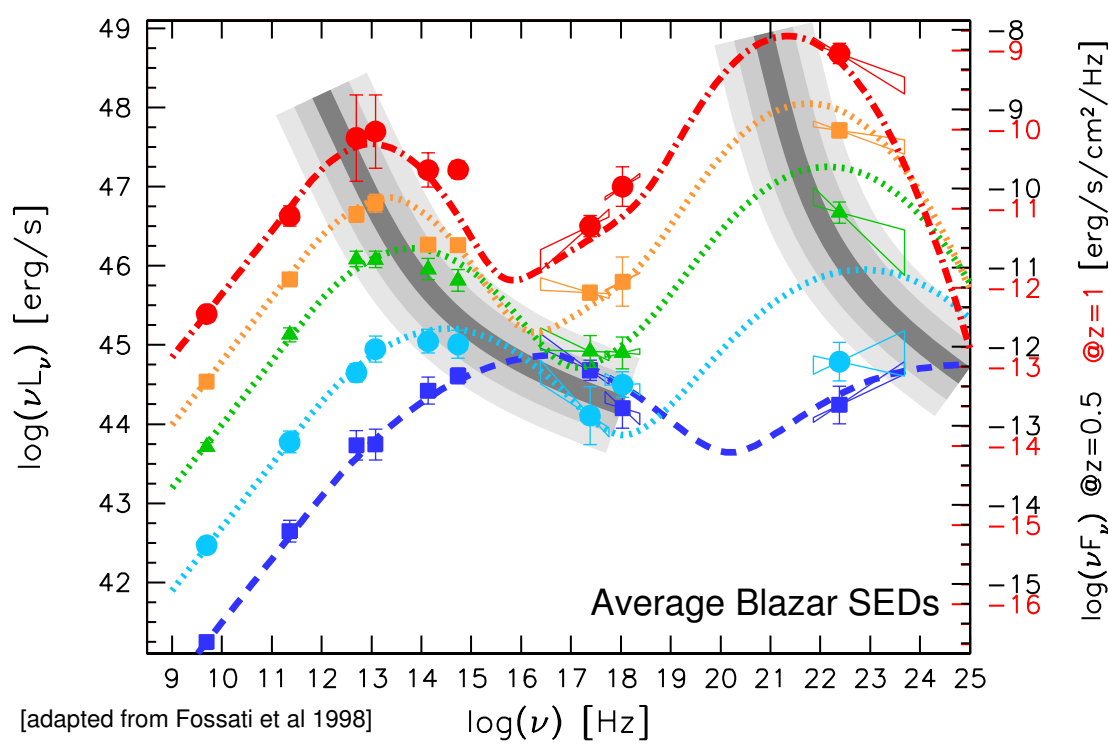

Figure 2: This is as close to an HR-type diagram our understanding of radio loud active galactic nuclei (AGN) has come: as the observed power of blazars, radio loud AGN with jets pointing close to the line of sight, increases, the peak frequency of both spectral components decreases and the dominance of the second component increases. This result [Fossati et al.(1998)] is being challenged by observations that carry the seed of a new and deeper understanding of the physics of extragalactic relativistic jets and their connection to accretion onto supermassive black holes

jets less aligned to the line of sight. Indeed, [Padovani et al.(2003)] found that new sources they identified modify the blazar sequence to an envelope, with the area below the blazar sequence populated with sources. Similar envelopes were found by [Antón \& Browne(2005)] and

[Nieppola et al.(2006)].

Challenges. [Caccianiga \& Marchã(2004)] found several low $L_{s}$ - low $v_{s}$ sources that, because they have a high core dominance $(R$, ratio of core and therefore beamed to extended and therefore isotropic radio emission), are not intrinsically bright sources at a larger jet angle. These sources challenge the sequence because (i) both intrinsically weak and intrinsically powerful jets can have similar $v_{s}$ and (ii) intrinsically weak jets can produce a wide range of $v_{s}$ from $\left(10^{12}-10^{18} \mathrm{~Hz}\right)$. Another challenge came from [Landt et al.(2008)] who showed that, contrary to what is anticipated by the sequence, high and low synchrotron peak frequency (HSP and LSP) BL Lacertae objects (BLs, blazars with emission line EW $W<5 \AA$ ) have similar extended radio power. These findings challenge the sequence, even after being extended to include the sources in the envelope as debeamed analogs of the blazar sequence sources.

\subsection{The case for a critical accretion rate in radio loud AGN}

[Narayan et al.(1997)] showed that at a dimensionless critical value of the accretion rate $\dot{m}_{\text {crit }}=$ $\dot{M}_{a c c} / \dot{M}_{E d d} \sim 10^{-3}-10^{-2}$, the accretion switches from an ADAF (advection dominated accretion flow) with accretion luminosity $L_{a c c} \propto \dot{m}^{2} L_{E d d}$, radiatively inefficient mode to a standard, radiatively efficient thin disk with $L_{a c c} \propto \dot{m} L_{E d d}$. This criticality point may be connected to the transition 

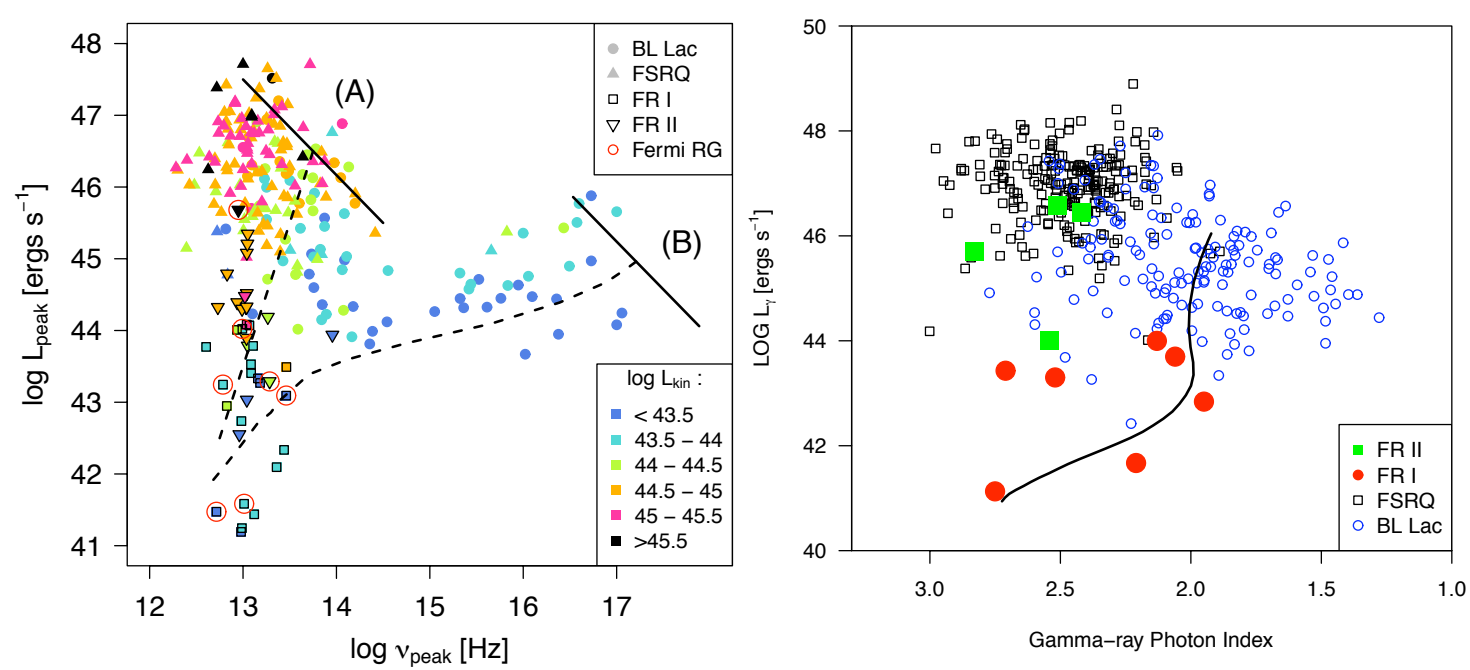

Figure 3: Left panel (from M11): The blazar sequence, which originally showed an anti-correlation between synchrotron peak luminosity and synchrotron peak frequency has been expanded into an 'envelope' with the addition of new observations and $R G$. The solid lines indicate a possible path of an aligned source as its $L_{k i n}$ increases. Track (A) shows the path of a synchrotron peak for a single speed jet, and (B) for a decelerating jet of the type hypothesized to exist in FRI sources as the jet orientation changes Right panel: The $\gamma$ envelope. This is figure 6 of [Abdo et al.(2010b)] rotated by $-\pi / 2$. The integrated $100 \mathrm{MeV}$ to $10 \mathrm{GeV}$ $\gamma$-ray luminosity of radio loud AGN is plotted as a function of their $\gamma$-ray photon index (used here as a proxy for the IC peak energy: the harder the photon index, the higher the IC peak energy). The solid line is the debeaming track of the decelerating jet model used also in the left panel of this figure. Note that in both the synchrotron and the $\gamma$ ray envelopes there are no high luminosity - high peak energy sources.

from FR I to FR II RG: the level of the low frequency extended radio emission (coming mostly from the radio lobes and considered to be isotropic) that separates FR I and FR II RG, has been shown to be a function of the host galaxy optical magnitude [Ledlow \& Owen(1996)]; the division between FR I and FR II is at higher radio luminosities for brighter galaxies. [Ghisellini \& Celotti(2001)] argued that this division can be casted as a division in terms of the fraction of the Eddington luminosity carried by the jet: jets with kinetic luminosity $L_{k i n}$ less than $\sim \dot{m}_{c r i t} L_{E d d}$ give rise to FR I RG, while jets with $L_{k i n} \gtrsim \dot{m}_{c r i t} L_{E d d}$ are predominantly FR II sources. Interestingly, and in agreement with the unification scheme, [Ghisellini \& Tavecchio(2008)] and [Ghisellini et al.(2009)] find that the same dichotomy applies to separating BLs and FSRQ, the aligned versions of FR I and FR II respectively.

\section{Current work}

\subsection{The unification of radio loud AGN and its relation to accretion}

To address the issue of the radio loud AGN unification, [Meyer et al.(2011)], (heretofore M11) formed the largest possible sample of radio loud AGN for which enough data exist to determine $v_{s}$ and the synchrotron peak luminosity $L_{S}$, as well as the extended low frequency radio emission 
$L_{e x t}$, which is a proxy for the jet kinetic power $L_{k i n}$ [Bîrzan et al.(2008), Cavagnolo et al.(2010)]. The emerging picture suggests that the population of extragalactic jets can be described in terms of two families. The first is that of weak jets characterized by velocity profiles and weak or absent broad emission lines. HSPs $\left(v_{s} \gtrsim 10^{16} \mathrm{~Hz}\right)$, ISPs $\left(10^{14.5} \lesssim v_{s} \lesssim 10^{16} \mathrm{~Hz}\right)$, and FR I RG belong to this family. On the basis of having similar $L_{k i n}$ with HSPs and FR I RG, the ISP sources were argued to be somewhat unaligned HSPs. The second family is that of more powerful jets characterized by a single Lorentz factor emitting plasma and, in most cases, stronger broad emission lines. Interestingly, the two families divide roughly at $L_{k i n} \sim 10^{44.5} \mathrm{erg} \mathrm{s}^{-1}$, which for a $M=10^{9} M_{\odot}$, corresponds to $L_{k i n} \sim 2.3 \times 10^{-3} L_{E d d, 9}$, similar to the $\dot{m}_{\text {crit }}$ of [Narayan et al.(1997)].

\subsubsection{Using aligned jets to infer the change of jet properties with jet power.}

The idea here is that different, physically motivated scenarios for how the properties of the jet change with $L_{k i n}$ will give us different tracks for the aligned sources on the $v_{\text {peak }}-L_{\text {peak }}$ synchrotron and IC diagrams. Comparing these models to the aligned sources (as inferred from their location on the $v_{\text {peak }}-L_{\text {peak }}$ diagram and their core to extended radio flux ratio) of M11 and its upcoming extensions along with data on the superluminal speeds for these sources [Lister et al.(2009)], we can determine the scaling of the main jet physical parameters with jet power and accretion environment. There are two cases that can be examined: $(i)$ an one-to-one correspondence between jet and accretion power, $L_{k i n} \propto L_{a c c}=\dot{m} L_{E d d}$, (ii) a range jet powers for a given accretion power.

Radiatively inefficient accretion. Let us follow a radio loud AGN of black hole mass $M$, with its jet axis aligned to the line of sight, as $L_{k i n}$ increases. Let the AGN accrete at a rate $\dot{m}<$ $\dot{m}_{c r i t}$. Following [Narayan et al.(1997)], we assume that the accretion is inefficient, the source does not exhibit strong broad lines, and is classified as a BL. The radiating electrons of these weak jets produce $\gamma$-rays emission by up-scattering synchrotron photons (synchrotron self Compton SSC) [Bloom \& Marscher(1996)]. As $L_{k i n} / L_{E d d}$ increases, the source shifts on the $v_{s}-L_{s}$ plot to delineate the aligned sequence track for inefficient accretion. In general we expect that as $L_{k i n}$ increases, $L_{s}$ increases and $v_{s}$ decreases due to increasing radiative cooling. The slope, however, of this track depends on the scaling of the different physical parameters with $L_{k i n}$. The particular aligned sequence (solid line B in Figure 3) for inefficient accretors results from jets that the electron power and Poynting flux scale with $L_{k i n}$, as is adopted for GRBs [Piran(2004)].

Radiatively efficient accretion. If $\dot{m} \gtrsim \dot{m}_{c r}$, accretion is efficient, characterized by strong emission from an accretion disk in the form of the well known optical-UV big blue bump (BBB) on which broad emission lines are superimposed, as well as IR emission from a molecular torus feeding the accretion disk. These will be visible only when the beamed synchrotron emission at these wavelengths does not dominate. The most easily detected of these components is the broad line emission. In this environment, these powerful jets produce $\gamma$-rays emission most probably by up-scattering external photons, either from the broad line region [Sikora et al.(1994)], or, if the emission takes place further out (e.g. [Agudo et al.(2011)]), form the molecular torus [Arbeiter et al.(2002)]. Here we again anticipate that due to increasing cooling with increasing $L_{k i n}$, as $L_{k i n}$ increases, $L_{s}$ will increase and $v_{s}$ will decrease.

The particular aligned sequence (solid line A) plotted in Figure 3 for efficient accretors results from jets for which $L_{k i n}$ scales with the jet Lorentz factor, as VLBI data suggest [Kharb et al.(2010)]. In this case, and if the comoving energy density of electrons and magnetic field remain invariant it 
can be shown that $L_{s} \propto v_{s}^{-4 / 3}$. Similarly to the inefficient branch, lines of different slope are anticipated for other scenarios in which cooling increases with increasing $L_{k i n}$. The EW $W$ of the broad lines for aligned sources is in general expected to increase along the efficient accretion track as $L_{k i n}$ increases. The reason for this is that although both the luminosity of the broad line region $L_{B L R}$ and $L_{s}$ increase as $L_{k i n}$ increases, because $v_{s}$ decreases, the ratio of $L_{B L R}$ to the synchrotron luminosity at optical-UV energies $L_{s, o p t-U V}$ increases, and, therefore, $W$ increases. This is in qualitative agreement with the left panel of Figure 3, where we see that along the solid line B, at small $L_{k i n}$ we encounter more BLs while at high $L_{k i n}$, we encounter mostly FSRQs. A detailed comparison of model predictions to observations will constrain the way the jet parameters scale with jet power.

\subsubsection{Deriving velocity profiles from the envelope of the unaligned sources}

Radiatively efficient accretion. The $v_{s}-L_{s}$ tracks for sources that gradually become more unaligned are related to the velocity profiles that characterize the source. For efficient accretors, where we assume a single velocity flow, sources of a given $L_{k i n}$ will follow a track of the form $L_{s} \propto v_{s}^{1 / 4}$ (broken line at the left of Figure 3). As $\theta$ increases, the broad line EW $W$ increases, due to the de-beaming of the synchrotron emission, as $W \propto v_{s}^{-(3+\alpha)}$, where $\alpha$ is the synchrotron SED spectral index at $v>v_{s}$. This increase of $W$ with increasing $\theta$ ceases when the de-beaming lowers the synchrotron level below that of the BBB. At large $\theta$ the source is classified as an FR II broad line RG, in broad agreement with the results of M11, as can be seen in the left panel of Figure 3. We also expect that the VLBI superluminal speed will in general decrease along the debeaming track (we may still find a few low $\beta_{a p p}$ sources that are very well aligned at $\theta<1 / \Gamma$ ).

Radiatively inefficient accretion. Similarly, for inefficient accretion, where (see §1.3) the flow exhibits velocity gradients in the form of a decelerating flow [Georganopoulos \& Kazanas(2003)] and/or a fast spine-slow sheath [Ghisellini et al.(2005)], the faster part of the jet carries the most energetic electrons and produces the highest energy synchrotron emission, which is also the mostly beamed. For this reason, as $\theta$ increases, $v_{s}$ decreases rapidly (broken line at the right of Figure 3 ), with the exact shape of the track depending on the deceleration profile. In this scenario, ISP sources $\left(10^{14.5} \mathrm{~Hz} \lesssim v_{s} \lesssim 10^{16}, \mathrm{~Hz}\right)$ are relatively unaligned HSP and are expected to have similar $L_{k i n}$ with their better aligned HSP siblings, as is seen to be the case in Figure 3. For larger $\theta$ the source becomes an FR I RG, also in qualitative agreement with observations shown in Figure 3.

\subsubsection{Constraints from the envelope at $\gamma$-ray energies}

Fermi has seen a manifestation of the blazar envelope, but so far it has been unnoticed. The right panel of Figure 3 is Figure 6 of [Abdo et al.(2010)], simply rotated by $-\pi / 2$. Using the $\gamma$-ray photon index $\Gamma$ as a proxy for the peak frequency $v_{\gamma}$ of the $\gamma$-ray component and the $100 \mathrm{MeV}-10$ $\mathrm{GeV}$ luminosity as a proxy of the $\gamma$-ray peak luminosity $L_{\gamma}$, we see a situation that has important similarities with the synchrotron envelope (left panel of Figure 3):

- As in the synchrotron case, there is a forbidden zone with no high luminosity hard sources.

- The FSRQs strongly cluster in the high $L_{\gamma}$-soft part of the plot and most BLs occupy the low $L_{\gamma}$-hard part of the plot, with a good fraction of them overlapping with the FSRQs.

- FR IIs and FSRQs have similar $\Gamma$, while FR Is appear, on the average, steeper than BLs.

Although the number of RG detected so far is small, the fact that FR II RG lie below FSRQs suggests that the flow of powerful sources is characterized by a single Lorentz factor. Also, the 
fact that FR Is are not only dimmer than BLs, but also steeper, suggests that these sources are characterised by velocity gradients. We present a case of this in the right panel of Figure 3 where we plot the IC track of the same decelerating jet shown in the left panel of Figure 3. An additional qualitative argument in favor of velocity profiles in weak sources is that we do not see hard low luminosity sources (not an obvious selection effect): when unaligned, the high energy part of the SED is beamed away from the line of sight and the SED peak is produced by the much lower energy electrons found in the slow part of the flow. This is also the case in the synchrotron envelope: no RG peaking at high frequencies, as would be expected if HSPs were characterized by a single velocity.

\section{References}

[Abdo et al.(2010)] Abdo, A. A., et al. 2010, ApJ, 716, 30

[Abdo et al.(2010b)] Abdo, A. A., et al. 2010, ApJ, 720, 912

[Agudo et al.(2011)] Agudo, I., et al. 2011, ApJL, 726, L13

[Antón \& Browne(2005)] Antón, S., \& Browne, I. W. A. 2005, MNRAS, 356, 225

[Arbeiter et al.(2002)] Arbeiter, C., Pohl, M., \& Schlickeiser, R. 2002, A\&A, 386, 415

[Begelman et al.(2008)] Begelman, M. C., Rossi, E. M., \& Armitage, P. J. 2008, MNRAS, 387, 1649

[Bîrzan et al.(2008)] Bîrzan, L., McNamara, B. R., Nulsen, P. E. J., Carilli, C. L., \& Wise, M. W. 2008, ApJ, 686, 859

[Blandford \& Znajek(1977)] Blandford, R. D., \& Znajek, R. L. 1977, MNRAS, 179, 433

[Blandford \& Payne(1982)] Blandford, R. D., \& Payne, D. G. 1982, MNRAS, 199, 883

[Bloom \& Marscher(1996)] Bloom, S. D., \& Marscher, A. P. 1996, ApJ, 461, 657

[Böttcher(2007)] Böttcher, M. 2007, APSS, 309, 95

[Caccianiga \& Marchã(2004)] Caccianiga, A., \& Marchã, M. J. M. 2004, MNRAS, 348, 937

[Cavagnolo et al.(2010)] Cavagnolo, K. W., McNamara, B. R., Nulsen, P. E. J., Carilli, C. L., Jones, C., \& Bîrzan, L. 2010, ApJ, 720, 1066

[Chiaberge et al.(2000)] Chiaberge, M., Celotti, A., Capetti, A., \& Ghisellini, G. 2000, A\&A, 358, 104

[Fanaroff \& Riley(1974)] Fanaroff, B. L., \& Riley, J. M. 1974, MNRAS, 167, 31P

[Fernandes et al.(2011)] Fernandes, C. A. C., et al. 2011, MNRAS, 411, 1909

[Fossati et al.(1998)] Fossati, G., Maraschi, L., Celotti, A., Comastri, A., \& Ghisellini, G. 1998, MNRAS, 299,433

[Georganopoulos \& Marscher(1998)] Georganopoulos, M., \& Marscher, A. P. 1998, ApJ, 506, 621

[Georganopoulos \& Kazanas(2003)] Georganopoulos, M., \& Kazanas, D. 2003, ApJL, 594, L27

[Ghisellini et al.(1998)] Ghisellini, G., Celotti, A., Fossati, G., Maraschi, L., \& Comastri, A. 1998, MNRAS, 301, 451

[Ghisellini \& Celotti(2001)] Ghisellini, G., \& Celotti, A. 2001, A\&A, 379, L1

[Ghisellini et al.(2005)] Ghisellini, G., Tavecchio, F., \& Chiaberge, M. 2005, A\&A, 432, 401 
[Ghisellini \& Tavecchio(2008)] Ghisellini, G., \& Tavecchio, F. 2008, MNRAS, 387, 1669

[Ghisellini et al.(2009)] Ghisellini, G., Maraschi, L., \& Tavecchio, F. 2009, MNRAS, 396, L105

[Ghisellini et al.(2011)] Ghisellini, G., Tavecchio, F., Foschini, L., \& Ghirlanda, G. 2011, MNRAS, 414, 2674

[Kharb et al.(2010)] Kharb, P., Lister, M. L., \& Cooper, N. J. 2010, ApJ, 710, 764

[Landt et al.(2006)] Landt, H., Perlman, E. S., \& Padovani, P. 2006, ApJ, 637, 183

[Landt et al.(2008)] Landt, H., Padovani, P., Giommi, P., Perri, M., \& Cheung, C. C. 2008, ApJ, 676, 87

[Ledlow \& Owen(1996)] Ledlow, M. J., \& Owen, F. N. 1996, AJ, 112, 9

[Lister et al.(2009)] Lister, M. L., et al. 2009, AJ, 138, 1874

[Maraschi et al.(2008)] Maraschi, L., Foschini, L., Ghisellini, G., Tavecchio, F., \& Sambruna, R. M. 2008, MNRAS, 391, 1981

[McNamara \& Nulsen(2007)] McNamara, B. R., \& Nulsen, P. E. J. 2007, ARA\&A, 45, 117

[Meyer et al.(2011)] Meyer, E. T., Fossati, G., Georganopoulos, M., \& Lister, M. L. 2011, ApJ, in press, also in arXiv:1107.5105

[Narayan et al.(1997)] Narayan, R., Garcia, M. R., \& McClintock, J. E. 1997, ApJL, 478, L79

[Nieppola et al.(2006)] Nieppola, E., Tornikoski, M., \& Valtaoja, E. 2006, A\&A, 445, 441

[Padovani et al.(1997)] Padovani, P., Giommi, P., \& Fiore, F. 1997, MNRAS, 284, 569

[Padovani et al.(2003)] Padovani, P., Perlman, E. S., Landt, H., Giommi, P., \& Perri, M. 2003, ApJ, 588, 128

[Padovani(2007)] Padovani, P. 2007, Ap\&SS, 309, 63

[Perlman et al.(1998)] Perlman, E. S., Padovani, P., Giommi, P., Sambruna, R., Jones, L. R., Tzioumis, A., \& Reynolds, J. 1998, AJj, 115, 1253

[Piner et al.(2010)] Piner, B. G., Pant, N., \& Edwards, P. G. 2010, ApJ, 723, 1150

[Piran(2004)] Piran, T. 2004, Reviews of Modern Physics, 76, 1143

[Scarpa \& Falomo(1997)] Scarpa, R., \& Falomo, R. 1997, A\&A, 325, 109

[Sikora et al.(1994)] Sikora, M., Begelman, M. C., \& Rees, M. J. 1994, ApJ, 421, 153

[Sikora et al.(2007)] Sikora, M., Stawarz, Ł., \& Lasota, J.-P. 2007, ApJ, 658, 815

[Stickel et al.(1991)] Stickel, M., Padovani, P., Urry, C. M., Fried, J. W., \& Kuehr, H. 1991, ApJ, 374, 431

[Stocke et al.(2011)] Stocke, J. T., Danforth, C. W., \& Perlman, E. S. 2011, ApJ, 732, 113

[Trussoni et al.(2003)] Trussoni, E., Capetti, A., Celotti, A., Chiaberge, M., \& Feretti, L. 2003, A\&A, 403, 889

[Urry \& Padovani(1995)] Urry, C. M., \& Padovani, P. 1995, PASP, 107, 803

[Vermeulen et al.(1995)] Vermeulen, R. C., Ogle, P. M., Tran, H. D., Browne, I. W. A., Cohen, M. H., Readhead, A. C. S., Taylor, G. B., \& Goodrich, R. W. 1995, ApJL, 452, L5 